Research article

\title{
Comparison of various characteristics of women who do and do not attend for breast cancer screening
}

\author{
Emily Banks*, Valerie Beral ${ }^{*}$, Rebecca Cameron*, Ann Hogg ${ }^{*}$, Nicola Langley ${ }^{*}$, Isobel Barnes*, \\ Diana Bull*, Gillian Reeves ${ }^{\star}$, Ruth English ${ }^{\dagger}$, Sarah Taylor ${ }^{\ddagger}$, Jon Elliman ${ }^{\S}$ and Carole Lole Harris ${ }^{\Uparrow}$
}

\author{
*Imperial Cancer Research Fund Cancer Epidemiology Unit, University of Oxford, Radcliffe Infirmary, Oxford, UK \\ ${ }^{+}$Breast Care Unit, Oxford Radcliffe Hospital NHS Trust, Oxford, UK \\ *Breast Care Unit, Princess Margaret Hospital, Swindon, UK \\ \$Kingswood Surgery, Park North, Swindon, UK \\ IDeer Park Surgery, Witney, UK
}

Correspondence: Emily Banks, Imperial Cancer Research Fund Cancer Epidemiology Unit, University of Oxford Gibson Building, Radcliffe Infirmary, Oxford OX2 6HE, UK. Tel: +44 (0)1865 311 933; fax: +44 (0)1865 310 545; e-mail: bankse@icrf.icnet.uk

Received: 14 June 2001

Revisions requested: 9 August 2001

Revisions received: 24 August 2001

Accepted: 19 September 2001

Published: 6 November 2001
Breast Cancer Res 2002, 4:R1

(C) 2002 Banks et al., licensee BioMed Central Ltd

(Print ISSN 1465-5411; Online ISSN 1465-542X)

\begin{abstract}
Background: Information regarding the characteristics and health of women who do and do not attend for breast cancer screening is limited and representative data are difficult to obtain.

Methods: Information on age, deprivation and prescriptions for various medications was obtained for all women at two UK general practices who were invited to breast cancer screening through the National Health Service Breast Screening Programme. The characteristics of women who attended and did not attend screening were compared.

Results: Of the 1064 women invited to screening from the two practices, 882 (83\%) attended screening. Screening attenders were of a similar age to non-attenders but came from significantly less deprived areas (30\% of attenders versus $50 \%$ of non-attenders came from the most deprived areas, $P<0.0001)$ and were more likely to have a current prescription for hormone replacement therapy (32\% versus $19 \%, P<0.0001$ ). No significant differences in recent prescriptions of medication for hypertension, heart disease, hypercholesterolaemia, diabetes mellitus, asthma, thyroid disease or depression/anxiety were observed between attenders and non-attenders.

Conclusion: Women who attend the National Health Service Breast Screening Programme come from less deprived areas and are more likely to have a current prescription for hormone replacement therapy than non-attenders, but do not differ in terms of age or recent prescriptions for various other medications.
\end{abstract}

Keywords: attendance, breast screening, mammography, The Million Women Study, United Kingdom

\section{Introduction}

The effectiveness of breast cancer screening programmes in reducing mortality from breast cancer is dependent on maintaining adequate attendance at screening among eligible women. Information regarding the general characteristics and health of women who do and do not attend for breast cancer screening is limited and representative data are difficult to obtain. This paper compares the character- istics of women who do and do not attend for breast cancer screening through the National Health Service Breast Screening Programme (NHSBSP), in terms of age, deprivation and prescriptions for a variety of commonly used medications. It also assesses the characteristics of women from the same population who agreed to participate in the Million Women Study, which recruits women through the NHSBSP.

HRT = hormone replacement therapy; NHSBSP = National Health Service Breast Screening Programme. 


\section{Materials and methods}

This study was based at the Deer Park Medical Centre in Witney, Oxfordshire (UK) and the Kingswood Surgery in Swindon, Wiltshire (UK). These practices were chosen to cover a relatively broad social class spectrum, from the comparatively affluent, semi-rural practice in Witney to the less affluent inner-city practice in Swindon. A computerised record of prescriptions has been kept at the Witney practice since 1992 and at the Swindon practice since 1994. All women invited to attend the NHSBSP from these general practices during 1998 were matched to the practices' computerised prescription records. All medications prescribed in the six months before screening were noted. Additional details were gathered on prescriptions for hormone replacement therapy (HRT), including date of first and most recent prescription of HRT.

The Million Women Study is a population-based multicentre prospective study in the UK aiming to investigate the effects of HRT on health. The methods are described in detail elsewhere [1]. Women invited to screening at participating NHS breast screening centres receive a questionnaire for the Million Women Study accompanying their invitation and are asked to join the study by returning the recruitment form when they attend for screening. Women participating in the Million Women Study from the Witney and Swindon practices were identified by matching the list of study participants against the list of women invited to breast cancer screening. Prescription data from women not participating in the Million Women Study were extracted anonymously under the supervision of practice staff. Ethical approval for the study was given by the Central Oxfordshire Research Ethics Committee and the Swindon Research Ethics Committee.

\section{Ascertainment and classification of variables}

Attendance at screening was defined as having undergone mammographic screening for breast cancer at the respective screening centre within the six month period following the screening invitation. The Townsend score, based on each participant's postcode, was used as a measure of deprivation; this score is obtained using information regarding unemployment, overcrowding and car and home ownership within each relevant enumeration district [2]. In the general population, this score usually ranges from -3 to +3 , and a higher score represents worse deprivation.

Ever use of HRT was considered to be the presence of more than one prescription for oral or transdermal HRT in the prescription record. Women who had received one isolated prescription (which had not necessarily been dispensed or taken) were therefore considered non-users. A woman was considered to be a current user of HRT if her last prescription date was within six months before her screen date, since a single prescription can provide med-
Table 1

\begin{tabular}{lcc} 
Study population & & \\
\hline Attendance status & $\mathrm{n}$ & $\%$ \\
\hline Total invited to screening & 1133 & 100 \\
Exclusions & & \\
$\quad$ Recently screened & 23 & 2 \\
$\quad$ Deferred attendance & 18 & 2 \\
$\quad$ Ineligible on medical grounds & 17 & 2 \\
$\quad$ Moved away & 11 & 1 \\
Total eligible & 1064 & 94 \\
\hline
\end{tabular}

ication for this period of time. Other medications on the prescription record were classified by a medically qualified researcher (Emily Banks) according to the following broad treatment categories: anti-hypertensive medications (e.g. relevant beta-blockers, diuretics, angiotensin converting enzyme inhibitors, calcium channel blockers and others); heart disease medications (e.g. digoxin, glyceryl trinitrate, etc); lipid lowering medications (e.g. statins, clofibrate, etc.); anti-diabetic medications (e.g. insulin, oral hypoglycaemics); asthma medications (e.g. bronchodilators, inhaled steroids, etc.); thyroid medications (e.g. thyroxine, carbimazole, etc.); and medications for depression/anxiety (e.g. anti-depressants, anxiolytics). Medications not corresponding to these categories were not coded. Medications that were not specific to one class of disorders were coded only in one treatment category, hierarchically as written above. Current treatment was considered to be a prescription within six months prior to the screening date.

\section{Analysis}

Women who had left the practice, either according to the breast screening centre records or the practice records, were excluded from the analyses (Table 1). Women who contacted the breast screening centre and indicated that they had recently been screened elsewhere or who were deemed ineligible on medical grounds were also excluded, as were those who deferred attendance at screening.

The Student's $t$-test was used for comparisons of mean age and deprivation between screening attenders and nonattenders. The same comparisons were also made among women who attended screening, comparing those who participated in the Million Women Study with those who did not. For comparison of prescription record medication use between groups, simple chi-squared tests were used.

\section{Results}

Of the 1064 eligible women who were invited to screening, $882(83 \%)$ attended screening. Among those who attended, $570(65 \%)$ filled in the Million Women Study 


\begin{tabular}{|c|c|c|c|c|c|c|c|}
\hline & \multirow[b]{4}{*}{$\begin{array}{c}\text { All eligible women } \\
\mathrm{n}(\%)\end{array}$} & \multirow{2}{*}{\multicolumn{3}{|c|}{ Attended screening }} & \multirow{4}{*}{$\begin{array}{l}\text { Did not attend } \\
\text { screening } \\
\\
\text { Total } \\
\mathrm{n}(\%)\end{array}$} & \multicolumn{2}{|c|}{$t$-test $-P$ value } \\
\hline & & & & & & Overall & $\begin{array}{l}\text { Among } \\
\text { screened }\end{array}$ \\
\hline & & \multirow[b]{2}{*}{$\begin{array}{l}\text { Total } \\
\text { n (\%) }\end{array}$} & \multicolumn{2}{|c|}{ Million Women Study } & & \multirow{2}{*}{$\begin{array}{l}\text { Screened } \\
\text { vs not } \\
\text { screened }\end{array}$} & \multirow{2}{*}{$\begin{array}{c}\text { MWS } \\
\text { participant } \\
\text { vs } \\
\text { non-participant }\end{array}$} \\
\hline & & & $\begin{array}{l}\text { Participant } \\
\text { n (\%) }\end{array}$ & $\begin{array}{c}\text { Non-participant } \\
\mathrm{n}(\%)\end{array}$ & & & \\
\hline \multicolumn{8}{|l|}{ Age } \\
\hline $49-54$ & 353 (33) & $284(32)$ & 195 (34) & $89(29)$ & $69(38)$ & & \\
\hline $55-59$ & $286(27)$ & $239(27)$ & $147(26)$ & $92(29)$ & $47(26)$ & & \\
\hline $60+$ & $425(44)$ & $359(41)$ & $228(40)$ & $131(42)$ & $66(46)$ & & \\
\hline Mean age $(95 \% \mathrm{Cl})$ & $\begin{array}{c}57.4 \\
(57.1-57.7)\end{array}$ & $\begin{array}{c}57.5 \\
(57.2-57.8)\end{array}$ & $\begin{array}{c}57.4 \\
(57.0-57.8)\end{array}$ & $\begin{array}{c}57.7 \\
(57.2-58.2)\end{array}$ & $\begin{array}{c}57.1 \\
(56.4-57.8)\end{array}$ & 0.4 & 0.3 \\
\hline \multicolumn{8}{|l|}{ Deprivation level* } \\
\hline Least deprived & 348 (33) & $303(35)$ & $204(36)$ & $99(32)$ & $45(25)$ & & \\
\hline Medium deprived & 348 (33) & 304 (35) & 186 (33) & $118(38)$ & $44(25)$ & & \\
\hline Most deprived & 351 (34) & $263(30)$ & $170(31)$ & $93(30)$ & $88(50)$ & & \\
\hline Mean deprivation $(95 \% \mathrm{Cl})$ & $\begin{array}{c}0.42 \\
(0.21-0.63)\end{array}$ & $\begin{array}{c}0.22 \\
(-0.06-0.44)\end{array}$ & $\begin{array}{c}0.20 \\
(-0.09-0.48)\end{array}$ & $\begin{array}{c}0.26 \\
(-0.11-0.62)\end{array}$ & $\begin{array}{c}1.41 \\
(0.89-1.92)\end{array}$ & $<0.0001$ & 0.8 \\
\hline Total $^{\dagger}$ & $1064(100)$ & $882(83)$ & $570(54)$ & 312 (29) & $182(17)$ & & \\
\hline
\end{tabular}

$\mathrm{Cl}$, confidence interval. ${ }^{\star}$ Measured by Townsend score. A higher score corresponds to more deprivation. ${ }^{\dagger}$ Numbers do not always add up to total due to missing values.

questionnaire and gave signed permission for follow-up (Table 2).

\section{Age and deprivation}

The average age and age distribution of the attenders and non-attenders was similar (Table 2). Women who did not attend screening, however, came from more deprived areas than women who attended screening (50\% of nonattenders versus $30 \%$ of attenders came from the most deprived areas, $P<0.0001)$. Among women who attended screening, there was no significant difference between Million Women Study participants and non-participants in terms of age or level of deprivation (Table 2).

\section{Prescriptions for HRT}

Overall, $42 \%$ of women who attended screening had more than one prescription for HRT recorded at some time since the records started and $32 \%$ had prescription records consistent with current use of HRT, compared to $27 \%$ and $19 \%$, respectively, among women who did not attend screening $\left(\chi^{2}{ }_{1}=13.5, P<0.0001\right.$ and $\chi^{2}{ }_{1}=13.6$, $P<0.0001$, respectively; Fig. 1$)$.

Restricting the analysis to women who attended screening, statistical comparisons were possible between Million
Women Study participants and non-participants. 35\% of Million Women Study participants had prescription records compatible with current use of HRT, compared to $28 \%$ among women who attended screening but did not join the study $\left(\chi^{2}{ }_{1}=5.2, P=0.02\right)$. Ever use of HRT during the time covered by the prescription record was significantly more common among women participating in the Million Women Study, compared to women who attended screening but did not join the Million Women Study (45\% versus 35\%, $\left.\chi^{2}{ }_{1}=7.7, P=0.005\right)$.

The prevalence of prescriptions compatible with current use of HRT was $30 \%$ in the general practice population eligible for screening, 32\% among women who attended screening and $35 \%$ among women who attended screening and participated in the Million Women Study (Fig. 1).

\section{Current use of various other medications}

There was no significant difference between attenders and non-attenders with respect to the proportion that had recently been prescribed medications for hypertension $\left(\chi^{2}{ }_{1}=0.6, P=0.4\right)$, heart disease $\left(\chi^{2}{ }_{1}=3.4, P=0.07\right)$, cholesterol lowering $\left(\chi^{2}{ }_{1}=0.4, P=0.5\right)$, diabetes mellitus $\left(\chi^{2}{ }_{1}=1.9, P=0.2\right)$, asthma $\left(\chi^{2}{ }_{1}=3.5, P=0.06\right)$, thyroid disease $\left(\chi^{2}{ }_{1}=1.3, P=0.2\right)$ or depression/anxiety 


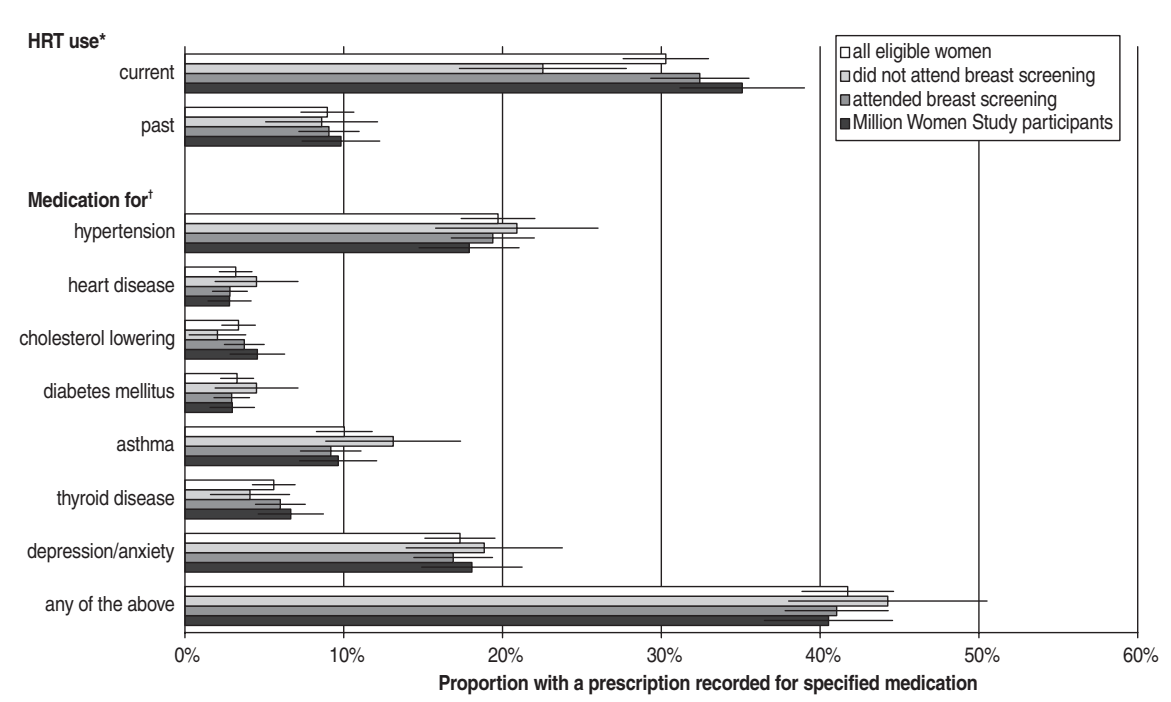

Prevalence of prescriptions for HRT and other medications according to screening attendance and participation in the Million Women Study. Horizontal bars indicate $95 \%$ confidence intervals. ${ }^{*}$ A prescription for HRT within the past six months is considered current use of HRT; a prescription for HRT on the prescription record but no prescription within the past six months is considered past use of HRT. ${ }^{\dagger}$ Any prescription within the past six months for medications for conditions listed here.

$\left(\chi^{2}{ }_{1}=0.002, P=0.9\right)$. In total, $44 \%$ of women who did not attend screening had prescriptions for at least one of these conditions, compared with $41 \%$ of women who attended screening $\left(\chi^{2}{ }_{1}=0.5, P=0.5\right.$; Fig. 1$)$.

There were no significant differences between Million Women Study participants and non-participants who attended screening with respect to the proportion that had recently been prescribed medications for hypertension $\left(\chi^{2}{ }_{1}=2.3, P=0.1\right)$, heart disease $\left(\chi^{2}{ }_{1}=0.004, P=0.9\right)$, cholesterol lowering $\left(\chi^{2}{ }_{1}=3.0, P=0.1\right)$, diabetes mellitus $\left(\chi^{2}{ }_{1}=0.01, P=0.9\right)$, asthma $\left(\chi^{2}{ }_{1}=0.4, P=0.5\right)$, thyroid disease $\left(\chi^{2}{ }_{1}=1.2, P=0.3\right)$, depression/anxiety $\left(\chi^{2}{ }_{1}=1.6\right.$, $P=0.2$ ) or with respect to the proportion of women prescribed some type of medication for at least one of these conditions $\left(\chi^{2}{ }_{1}=0.2, P=0.7 ;\right.$ Fig. 1$)$. There were no large differences between Million Women Study participants and the general population in terms of current prescriptions for these medications, nor were there any large differences between Million Women Study participants and the overall group of women who attended screening.

\section{Discussion}

These data indicate that women who attend for breast cancer screening are significantly more likely to come from less deprived areas than women who do not attend for screening, and are significantly more likely to have a prescription record compatible with current use of HRT. No significant differences between attenders and non-attenders were observed in terms of age or prescriptions for various other medications. Women who went on to participate in the Million Women Study were slightly more likely to be prescribed HRT but did not differ from non-participants in terms of age, deprivation level or use of other prescription medications.

The data presented here are consistent with previous reports in finding lower socio-economic status among women who do not attend for breast cancer screening compared to women who do attend [3-7]. Our study finds that $25 \%$ of women from the most deprived areas included in the study (88 of 351) did not attend screening, compared to $13 \%$ non-attendance among women from the medium deprived (44 of 348) and least deprived (45 of 348) areas. This problem is well recognised and attempts to improve uptake in deprived areas continue to be a priority for the NHSBSP.

Previous studies suggest that women who attend breast screening are less likely than non-attenders to have serious illness, but are more likely to have visited a physician recently and to have made use of other screening services [3,6-9]. One study found perceived health status to be similar in attenders and non-attenders [10] while another found that non-attenders had poorer perceived health status than attenders [8]. We did not observe significant differences between attenders and non-attenders in terms of recent prescriptions for common illnesses. Such prescriptions have been shown to agree well with self-reported treatment for various illnesses [11]. 
Prescriptions for HRT were significantly more common among women who attended screening than among those who did not attend. Around $70 \%$ of women who attend for screening at participating NHS breast screening centres take part in the Million Women Study [1]. The prevalence of current use of HRT was $30 \%$ in the eligible general practice population invited to screening, 32\% among women attending screening and $35 \%$ in Million Women Study participants. These findings are consistent with those of previous studies that have shown that women who take HRT are more likely to make use of various medical and screening services, including mammography, than women who do not use HRT $[8,12,13]$. Furthermore, the increasing awareness that women who use HRT are at a higher risk of breast cancer than non-users [14] may also encourage them to attend screening. Differences in the prevalence of use of HRT were greater between those who did and did not attend screening than between those who attended screening and did or did not participate in the Million Women Study.

Previous studies comparing attenders and non-attenders at screening have been based on interviews and postal questionnaires and have been hampered by the fact that women who do not attend breast cancer screening have a low response rate to surveys $[3-6,9,15,16]$. Many studies report response rates of $50 \%$ or less in non-attenders $[6,9,10,16]$. Other problems have arisen where the nonattenders have been a heterogeneous group composed of women who have been recently screened elsewhere and women who do not attend mammographic screening at all [9]. In our study, deprivation, screening and prescription record data were equally available for all women, and were independent of their subsequent attendance at screening. Women known to have declined screening because of being screened recently elsewhere were excluded.

The limitations of the data presented here should also be acknowledged. The study was conducted at two general practices in Southern England and the practices were selected to reflect different levels of deprivation in their respective populations. Women invited to screening from the inner-city Swindon practice were significantly more deprived than those invited from the semi-rural Witney practice (mean deprivation score 1.27 versus -1.83 , $P<0.0001)$. However, results may not be generalisable to the entire UK. For some variables there was limited power to ascertain differences between the groups, particularly in terms of prescriptions for uncommon conditions such as heart disease or diabetes. In addition, some of the women who did not attend for screening may have left the practice and would, therefore, not have had recent prescriptions for various medications, resulting in the prevalence of prescriptions appearing lower than is really the case in non-participants. Eleven women (1\%) were excluded from the study because they were known to have left the practice since invitations to breast screening had been sent out, but whether or not more had left the practice is not known.

\section{Conclusion}

In summary, women who attended screening came from less deprived areas and were more likely to use HRT than women who did not attend screening. Women who went on to take part in the Million Women Study did not differ substantially from the overall population of women who attended screening.

\section{Acknowledgements}

We thank the women who completed questionnaires for this study. We are grateful to Ms Angela Balkwill and the staff at the Deer Park and Kingswood practices, including Sr C Hamilton, Ms J Sissons, Dr R Hillman, Dr Z Mileusnic, Dr J Bentley, Dr P Mayes, Mr R Purdon, Ms V Thompson. We thank Ms J Webb and Ms J Green of the Swindon and Oxfordshire Breast Screening Services.

\section{References}

1. The Million Women Study Collaborative Group: The Million Women Study: design and characteristics of the study population. Breast Cancer Res 1999, 1:73-80.

2. Townsend $\mathrm{P}$, Phillimore $\mathrm{P}$, Beattie $\mathrm{A}$ : Health and deprivation: inequality and the North. London: Croom Helm; 1988.

3. Vernon SW, Laville EA, Jackson GL: Participation in breast screening programs: a review. Soc Sci Med 1990, 30:11071118.

4. Calle EE, Flanders WD, Thun MJ, Martin LM: Demographic predictors of mammography and pap smear screening in US women. Am J Pub Health 1993, 83:53-60.

5. Hurley SF, Huggins RM, Jolley DJ, Reading D: Recruitment activities and sociodemographic factors that predict attendance at a mammographic screening programme. Am J Pub Health 1994, 84:1655-1658.

6. Sutton S, Bickler G, Sancho Aldridge J, Saidi G: Prospective study of predictors of attendance for breast screening in inner London. J Epidemiol Community Health 1994, 48:65-73.

7. Maclean U, Sinfield D, Klein S, Harnden B: Women who decline breast screening. J Epidemiol Community Health 1984, 38:278283.

8. Lagerlund M, Sparén P, Thurfjell E, Ekbom A, Lambe M: Predictors of non-attendance in a population-based mammography screening programme; socio-demographic factors and aspects of health behaviour. Eur J Cancer Prev 2000, 9:25-33.

9. Aro AR, de Koning HJ, Absetz P, Schreck M: Psychological predictors of first attendance for organised mammography screening. J Med Screening 1999, 6 :82-88.

10. Hunt SM, Alexander F, Roberts MM: Attenders and non-attenders at a breast screening clinic: a comparative study. Public Health 1988, 102:3-10.

11. Banks E, Beral V, Cameron R, Hogg A, Langley N, Barnes I, Bull D, Elliman J, Lole Harris C: Agreement between general practice prescription data and self-reported use of hormone replacement therapy and treatment for various illnesses. J Epidemiol Biostatistics 2001, 6:357-363.

12. Lancaster T, Surman G, Lawrence M Mant D, Vessey M, Thorogood M, Yudkin $P$, Daly $E$ : Hormone replacement therapy: characteristics of users and non- users in a British general practice cohort identified through computerised prescribing records. J Epidemiol Community Health 1995, 49:389-394.

13. Rosenberg L, Shapiro S, Kaufman DW, Slone D, Miettinen OS, Stolley PD: Patterns and determinants of conjugated estrogen use. Am J Epidemiol 1979, 109:676-686.

14. Collaborative Group on Hormonal Factors in Breast Cancer: Breast cancer and hormone replacement therapy: collaborative reanalysis of data from $\mathbf{5 1}$ epidemiological studies of $\mathbf{5 2}$ 705 women with breast cancer and 108411 women without breast cancer. Lancet 1997, 350:1047-1059.

15. Schofield PE, Cockburn J, Hill DJ, Reading D: Encouraging attendance at a screening mammography programme: deter- 
minants of response to different recruitment strategies. J Med Screening 1994, 1:144-149.

16. Turnbull D, Irwig L, Simpson JM, Donnelly N, Mock P: A prospective cohort study investigating psychosocial predictors of attendance at a mobile breast screening service. Australian J Public Health 1995, 19:172-176. 